\title{
Distinct expression profile of IncRNA in endometrial carcinoma
}

\author{
JUAN XU ${ }^{1 *}$, YUJIA QIAN $^{1 *}$, MIN YE $^{2 *}$, ZIYI FU $^{3}$, XUEMEI JIA ${ }^{1}$, WENQU LI $^{1}$, PENGFEI XU $^{3}$, \\ MINGMING LV ${ }^{2}$, LEI HUANG ${ }^{4}$, LUYU WANG ${ }^{2}$, HONGJIE RUAN ${ }^{1}$ and JUAN LV ${ }^{1}$ \\ Departments of ${ }^{1}$ Obstetrics and Gynecology, and ${ }^{2}$ Breast Surgery; ${ }^{3}$ Nanjing Maternity and Child Health Medical Institute, \\ Affiliated Obstetrics and Gynecology Hospital, Nanjing Medical University, Nanjing 210004; ${ }^{4}$ First Affiliated Hospital, \\ Nanjing Medical University, Nanjing, Jiangsu 210029, P.R. China
}

Received May 11, 2016; Accepted September 12, 2016

DOI: $10.3892 /$ or.2016.5173

\begin{abstract}
Endometrial carcinoma (EC) is the most common malignancy in women. Dispite its prevalence, the prognosis of endometrial carcinoma still relies on conventional histological type, grade and invasion information. Its morbidity is still increasing and the outcome is very poor. To the best of our knowledge, hormonal imbalance and/or molecular genetic alterations are the main cause of EC. However, the alterations of lncRNAs which accounts for approximately $4 / 5$ of human transcripts are still poorly understood. In the present study, using the RiboArray ${ }^{\mathrm{TM}}$ Custom Array, we studied the expression profiles of IncRNA in EC as compared to normal endometrium (NE) to find potential core IncRNAs for the diagnosis of EC. We found the potential core lncRNA by GO, KEGG, IncRNA and mRNA co-expression network. The potential functional lncRNAs were further detected by qPCR to validate the microarray results. A total of 172 lncRNAs and 188 mRNAs were found to be differentially expressed between type I EC and the NE samples (fold change >1.5). qPCR validation showed good consistency with the microarray data. GO, pathway analysis, the IncRNA and mRNA co-expression network as well as the TCGA data revealed that $6 \operatorname{lncRNAs}$ (KIAA0087, RP11-501O2, FAM212B-AS1, LOC102723552, RP11-140I24 and RP11-600K151) may be the core regulators
\end{abstract}

Correspondence to: Dr Hongjie Ruan or Dr Juan Lv, Department of Obstetrics and Gynecology, Affiliated Obstetrics and Gynecology Hospital, Nanjing Medical University, 123 Mochou Road, Nanjing, Jiangsu 210004, P.R. China

E-mail: hongjie_ruan@126.com

E-mail: juanlv2015@yahoo.com

*Contributed equally

Abbreviations: EC, endometrial carcinoma; EEC, endometrioid carcinoma; lncRNA, long non-coding RNA; mRNA, messenger RNA; GAPDH, glyceradehyde 3-phosphate dehydrogenase; GO, Gene Ontology; KEGG, Kyoto Encyclopedia of Genes and Genomes; UCSC, University of California Santa Cruz

Key words: endometrial carcinoma, microarray, lncRNA, GO, KEGG, co-expression network of endometrial carcinogenesis. The potential core lncRNAs revealed by the mRNA and lncRNA co-expression network might be helpful to explore potential early diagnostic and therapeutic targets for EC.

\section{Introduction}

Endometrial carcinoma is the most frequent malignancy in female genital tract accounting for 1-2\% mortality rate of all cancer in the western world (1). According to the statistic data collected from 2000 to 2011 in China, the morbidity has increased by $3.7 \%$ every year (2). Lack of an early diagnostic marker and therapeutic targets is the main cause of EC deaths.

In general, EC is mainly classified into two major types (type I and type II). Type I ECs comprise $80 \%$ of new cases (3). They are histologically well and moderately differentiated, estrogen-dependent and less prone to be aggressive. Type II ECs are mostly low differentiated, estrogen-independent and with a tendency to recur, even at early stage. The hormonal imbalance and the genetic alterations are proved to be the main cause of EC $(4,5)$. Most of EC cases are diagnosed after menopause. The use of estrogen only replacement therapy and late menopause onset can increase the risk of EC. Genetic alteration, such as MLH1 (6), MSH2 (7), PMS2 (8), MSH6 (9), ARID1A (10), PTEN (11), KRAS2 (12), CTNNB1 (13), RB1 (11), TP53 (12), c-Kit (14) gene mutations or gene loss have been demonstrated to be associated with the increased risk of EC, ATR mutation in endometrioid carcinoma (EEC) is associated with aggressiveness of the EEC (15). Thus, we believe that new molecular-based subtype classification can help for the early diagnosis and treatment decision.

Recently, the non-coding RNAs, including miRNA, lncRNAs (longer than $200 \mathrm{bp}$, not encoding proteins, transcribed by RNA polymerase II) which account for approximately $4 / 5$ of all human transcripts are proved to be critical regulators of cancers, including EC. They are involved in nearly all of the metastatic processes of cancer, such as proliferation, invasion and metastasis through regulating the protein coding gene expression transcriptionally, post-transcriptionally or by functioning as a scaffold of the lncRNA, miRNA and protein interaction (16). Besides, a variety of lncRNAs have been found to be the prognostic marker of diseases, such as breast (17), lung (18) and ovarian cancer (19). 
Table I. Details of primer pairs used in analysis of lncRNA and mRNA expression by qRT-PCR.

\begin{tabular}{lll}
\hline Gene & \multicolumn{1}{c}{ Forward primer (5'-3') } & \multicolumn{1}{c}{ Reverse primer (5'-3') } \\
\hline KIAA0087 & TCGGGGGACCGAGAAATACT & CCAGTCCAAGAGAAGCAGCA \\
RP11-508N22 & TTAGCAGCACATGCTCACCA & GGTAAGTAGCTGGGCTGTGG \\
RP11-501O2 & ACGATGAGACTTGGTGCTGAA & TGGTCCTGTTTCTCGCTGAC \\
FAM212B-AS1 & CCAGAGAAGAAAGGCAGCGA & ACTCCTTCGCGTGTTCAGTT \\
LOC102723552 & ACGAGATGCAGAAGATTACGC & TGAAATCTTACCTGACTGCAGATT \\
RP11-140I24 & TCAGTCGTCTTCACGCTTCC & CAGATTAGCTGGCAGAGGGG \\
RP11-600K151 & AGGGCTCAGTAGATTTGCCC & AGGCAGCATCTCACCTAACG \\
ALCAM & CTGGAGTACAAGACAACCAAGG & GTCACCTGCTCTGTAGGATAG \\
POLR3E & TTTCAGTACCCTGTGCGTCC & TGGGCTTGATCTTGGCTGAG \\
TDRD10 & GGAGATCCTGTTGGAAAGCA & GCCAACATACACCTCTGTCTC \\
\hline
\end{tabular}

Thus, the exploration of lncRNA expression profile is essential for the discovery of early diagnostic markers and the potential therapeutic candidates. Notably, Zhai et al (20), reported the differentially lncRNA in randomly selected in EC samples and the adjacent non-tumor tissues. However, the mechanism of the type I EC and type II EC is quite different, the differential lncRNAs in type I EC and the normal endometrium (NE) from the uterine leiomyoma patients which will give us the important information of the potential early diagnostic marker is poorly understood. In the present study, we aimed to find the potential early diagnostic and therapeutic candidates of IncRNAs for EC. We first utilized the high throughput lncRNA microarray analysis to find the differentially expressed lncRNAs. Then, we further analyzed the lncRNA function by Gene Ontology (GO), Kyoto Encyclopedia of Genes and Genomes (KEGG), mRNA-lncRNA co-expression network as well as the TCGA database to find the potential core lncRNAs that may be involved in EC. This would be helpful for exploring the mechanism of EC development as well as for prediction of clinical outcomes.

\section{Materials and methods}

Preparation of samples. Samples of human endometrial carcinoma (EC) and the NE (from the patients with hysterectomy) were collected immediately after surgery at the Obstetrics and Gynecology Hospital, affiliated to Nanjing Medical University. Tissue samples were collected with the consent of the patients and approved by the Ethics Committees in the hospital. In addition, all samples were pathologically confirmed. All samples were placed in TRIzol reagent (Invitrogen, Carlsbad, CA, USA) and stored at $-80^{\circ} \mathrm{C}$. RNA for RT-PCR was extracted using the RNeasy Mini kit (Qiagen, Valencia, CA, USA) according to the manufacturer's protocol. RNA samples for microarray were extracted by Guangzhou RiboBio Co., Ltd., (Guangzhou, China). Total RNA $(1 \mu \mathrm{g})$ was reversely transcribed using the Thermo Scientific ReverseAid First Strand cDNA Synthesis kit (Thermo Fisher Scientific, Wilmington, DE, USA).

Microarray. RNA preparation and microarray hybridization were performed by Guangzhou RiboBio Co., Ltd., according to their manufacturer's standard protocols. Briefly, RNA was purified from total RNA after removal of rRNA. Approximately 17899 lncRNAs and 26363 mRNAs are detected using the IncRNA microarray.

Quantitative real-time ( $q R T)$ PCR. qRT-PCR was performed using SYBR-Green (Applied Biosystems, Shanghai, China) on an Applied Biosystems ViiA ${ }^{\mathrm{TM}} 7 \mathrm{DX}$ machine (Life Technologies, Carlsbad, CA, USA). Assays were run in triplicate or quadruplicate and values were normalized to glyceraldehyde 3-phosphate dehydrogenase (GAPDH). Primers for each gene and lncRNA were designed according to the NCBI online primer-blast tool (http://www.ncbi.nlm.nih.gov/tools/ primer-blast/) and sequences are listed in Table I. The PCR conditions were $95^{\circ} \mathrm{C}$ for $30 \mathrm{sec}$, followed by 40 cycles of $95^{\circ} \mathrm{C}$ for $5 \mathrm{sec}, 60^{\circ} \mathrm{C}$ for $31 \mathrm{sec}$.

Statistical analysis. The data were analyzed using the Microsoft Excel 2007 (Microsoft, Redmond, WA, USA). The values are presented as mean \pm SEM. Student's t-test was used for the comparison of two independent groups $(\mathrm{P}<0.05, \mathrm{P}<0.01$ and $\mathrm{P}<0.001)$.

\section{Results}

Clinical characteristics of patient samples. EC samples were collected from 12 patients and the control endometrium were collected from at least 18 patients who underwent hysterectomy after having been diagnosed with uterine leiomyoma. According to the clinicopathological study, all the patients are type I EC at stage I/II. Pathology analysis showed that all EC patients are $\mathrm{PR}^{+} ; \mathrm{ER}^{+} ; \mathrm{Ki}-67^{+}(30-80 \%)$ with middle/high differentiated tissue morphology.

lncRNA microarray profiling. To find the potential roles of lncRNA function in EC, we first examined the differentially expressed lncRNA by microarray profiling (Fig. 1). lncRNAs were collected from the authoritative database NCBI-GRCh38, ENA, VEGA, RNAcentral, HANAVA, ENSEMBL, RFAM (Fig. 1A). In the data, a total of 17899 lncRNA and 26363 mRNA were detected with 172 lncRNA and 188 mRNA differentially expressed between type I EC (stage I/II) and their relative control endometrium (fold $>1.5$ and $\mathrm{P}<0.05$ ) as 
Table II. Dysregulated lncRNAs in the EC compared with the NE filtered by a fold-change $>5.0$ and $\mathrm{P}<0.05$

\begin{tabular}{|c|c|c|c|c|c|}
\hline Transcript:seqname & Regulation & Ratio & Chromosome & Strand & Database \\
\hline URS0000781FB6:URS0000781FB6 & Downregulation & 47.28 & $\operatorname{chr} 13$ & - & RNAcentral \\
\hline ENST00000624452:RP1-41C23 & Downregulation & 61.15 & $\operatorname{chr} 17$ & - & HAVANA \\
\hline URS00004EDFF6:AC013461 & Downregulation & 89.16 & $\operatorname{chr} 2$ & - & VEGA \\
\hline $\begin{array}{l}\text { XR_243449;XR_243450;XR_243448: } \\
\text { RP11-7O141 }\end{array}$ & Downregulation & 33.50 & $\operatorname{chr} 16$ & + & NCBI_GRCh38 \\
\hline XR_430290:LOC102723552 & Downregulation & 11.26 & $\operatorname{chr} 20$ & - & NCBI_GRCh38 \\
\hline ENST00000624279:RP11-140I24 & Downregulation & 42.61 & $\operatorname{chr} 16$ & - & HAVANA \\
\hline XR_427960:TRDN & Downregulation & 19.50 & chr6 & - & NCBI_GRCh38 \\
\hline URS0000415D28:RP11-311B18 & Downregulation & 39.35 & $\operatorname{chr} 10$ & - & VEGA \\
\hline URS0000506C5B:AC003090 & Downregulation & 54.03 & $\operatorname{chr} 7$ & - & VEGA \\
\hline XR_426628:ANKRD13C & Downregulation & 27.04 & chr1 & - & NCBI_GRCh38 \\
\hline URS000034FDBD:RP11-508N22 & Downregulation & 45.24 & $\operatorname{chr} 10$ & + & VEGA \\
\hline XR_427604:LOC102724353 & Downregulation & 27.03 & $\operatorname{chr} 4$ & - & NCBI_GRCh38 \\
\hline NR_002319:PIPSL & Downregulation & 20.52 & $\operatorname{chr} 10$ & - & NCBI_GRCh38 \\
\hline URS00004A7EA2:CTD-2410N18 & Downregulation & 31.52 & $\operatorname{chr} 5$ & + & VEGA \\
\hline URS00001B388A:AC097517 & Downregulation & 15.44 & $\operatorname{chr} 2$ & - & VEGA \\
\hline ENST00000620339:KLF3-AS1 & Downregulation & 35.30 & $\operatorname{chr} 4$ & - & HAVANA \\
\hline URS00000000FD:AC113618 & Downregulation & 30.04 & $\operatorname{chr} 2$ & + & ENA \\
\hline URS0000258264:RP11-501O2 & Downregulation & 18.88 & $\operatorname{chr} 3$ & + & ENA \\
\hline NR_022006:KIAA0087 & Downregulation & 9.25 & $\operatorname{chr} 7$ & - & NCBI_GRCh38 \\
\hline NR_039986:RP11-600K151 & Downregulation & 18.06 & chr8 & - & NCBI_GRCh38 \\
\hline NR_038951;NR_038952:FAM212B-AS1 & Downregulation & 51.75 & $\operatorname{chr} 1$ & + & NCBI_GRCh38 \\
\hline URS000049614A:RP11-140I24 & Downregulation & 10.28 & $\operatorname{chr} 16$ & + & ENA \\
\hline URS000058EAF9:RP11-680F8 & Downregulation & 8.16 & $\operatorname{chr} 15$ & + & ENA \\
\hline URS000041DE60:RP1-35C21 & Upregulation & 12.36 & $\operatorname{chr} 1$ & + & ENA \\
\hline URS000075B1F5:ROR1-AS1 & Upregulation & 10.60 & $\operatorname{chr} 1$ & - & RNAcentral \\
\hline NR_029671:MIR125B1 & Upregulation & 21.10 & $\operatorname{chr} 11$ & - & NCBI_GRCh38 \\
\hline URS00004B2E02:RP11-573J24 & Upregulation & 18.64 & chr8 & + & ENA \\
\hline URS00005369A5:RP11-474P2 & Upregulation & 32.39 & $\operatorname{chr} 12$ & - & VEGA \\
\hline URS00001CE0A9:AC098828 & Upregulation & 15.07 & $\operatorname{chr} 2$ & - & VEGA \\
\hline XR_243832:RP11-856M71 & Upregulation & 78.22 & $\operatorname{chr} 18$ & + & NCBI_GRCh38 \\
\hline ENST00000623186:RP11-449M6 & Upregulation & 19.09 & chr8 & + & HAVANA \\
\hline URS0000610163:RP11-539G18 & Upregulation & 7.00 & $\operatorname{chr} 4$ & + & ENA \\
\hline NR_027333:GPR158-AS1 & Upregulation & 7.01 & $\operatorname{chr} 10$ & - & NCBI_GRCh38 \\
\hline URS000032778C:RP11-1143G9 & Upregulation & 9.89 & $\operatorname{chr} 12$ & - & VEGA \\
\hline
\end{tabular}

shown in the scatter plot data (Fig. 1B and D) (the red dots indicate the differentially expressed lncRNA and mRNA with fold $>1.5$ and $\mathrm{P}<0.05$ ) and clustering data (Fig. $1 \mathrm{C}$ and $\mathrm{E}$; fold $>1.5$ and $\mathrm{P}<0.05)$. The detailed information of the differentially lncRNAs and mRNAs are presented in Tables II and III with the fold change $>5$ and $\mathrm{P}<0.05$.

Expression signature of dysregulated lncRNAs in EC compared to $N E$. To investigate the signature of the dysregulated lncRNAs in EC, we studied the dysregulated lncRNA classification, lengths and chromosome distribution (Fig. 2).
In the present study, the majority of the lncRNAs that were differentially expressed in EC were intergenic lncRNAs, antisense lncRNAs as well as the TEC (to be experimentally confirmed) lncRNAs (Fig. 2A). The lncRNA lengths are mainly between 400 and 64000 bp (Fig. 2B). The upregulated lncRNAs and the downregulated lncRNAs are distributed in different chromosomes (Fig. 2C and D).

GO and pathway analysis. To explore the potential function of the dysregulated lncRNAs in EC, we predicted the lncRNA target genes according to their chromosome location. In 

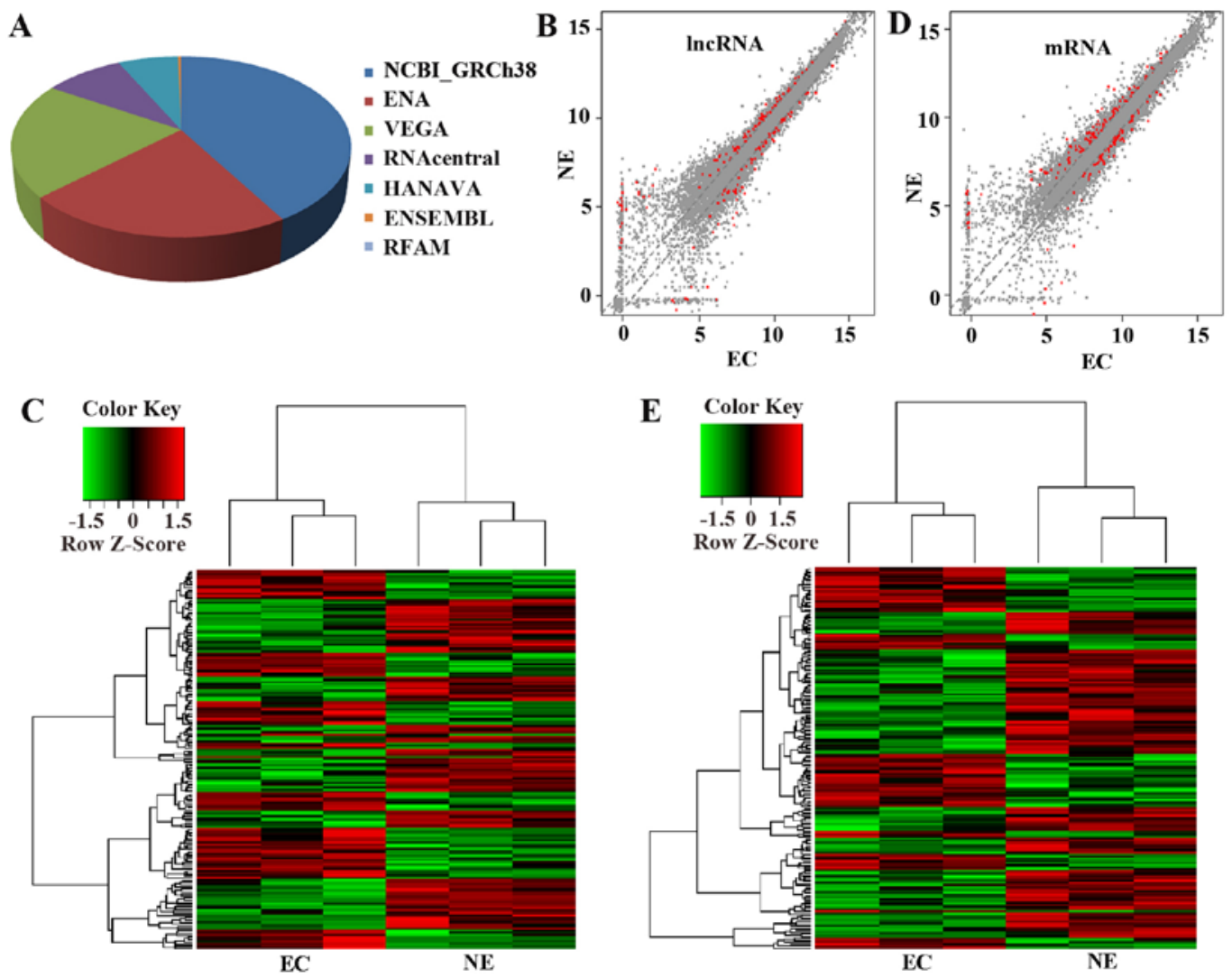

Figure 1. IncRNA and mRNA microarray profiling data of endometrial carcinoma (EC) and normal endometrium (NE). Using second generation microarray, $17899 \mathrm{lncRNAs}$ were identified. The pie chart shows the relative numbers of lncRNAs from the authoritative databases (A). The red points above the top green line indicate $>1.5$-fold upregulated lncRNAs (B) and mRNAs (D) in EC and the red points below the top green line indicate $>1.5$ fold-downregulated lncRNAs (B) and mRNAs (D) in EC (P<0.05). Hierarchical clustering shows a remarkable lncRNA (C) and mRNA (E) expression profile between EC and NE ( $>1.5$ fold and $\mathrm{P}<0.05$ ).

Table III. Dysregulated mRNAs in the EC compared with the $\mathrm{NE}$ filtered by a fold-change $>5.0$ and $\mathrm{P}<0.05$.

\begin{tabular}{llr}
\hline Name & \multicolumn{1}{c}{ Regulation } & \multicolumn{1}{c}{ Ratio } \\
\hline CLDN23 & Downregulation & 64.81788 \\
NEIL3 & Downregulation & 60.71055 \\
ALCAM & Downregulation & 27.00711 \\
TMEM68 & Downregulation & 68.43251 \\
POLR3E & Downregulation & 18.53199 \\
TDRD10 & Downregulation & 18.66308 \\
SELP & Downregulation & 40.2663 \\
FGFR2 & Downregulation & 15.83808 \\
DISP1 & Downregulation & 32.15958 \\
TBC1D22A & Downregulation & 27.66319 \\
GALK2 & Downregulation & 19.62079 \\
RTKN2 & Upregulation & 39.20362 \\
CADPS & Upregulation & 40.45117 \\
NLRP10 & Upregulation & 16.82022 \\
UPP1 & Upregulation & 5.53434 \\
EPC2 & Upregulation & 23.74738 \\
NXNL2 & Upregulation & 37.67642 \\
TXNL4B & Downregulation & 5.196587 \\
PCDH17 & Downregulation & 8.172227 \\
HSD17B2 & Downregulation & 7.949501 \\
LRRC1 & Downregulation & 5.796955 \\
STK25 & Downregulation & 5.488636 \\
\hline
\end{tabular}

addition, we carried out GO and pathway analysis of these lncRNAs. The Gene Ontology consortium which incorporate many databases and developed three ontologies (biological processes, cellular components and molecular functions) (http://www.geneontology.org) revealed that numerous biological processes, cellular components and molecular function are altered in EC compared to control (Fig. 3A-C). To find which pathways are altered, we also mapped the genes to KEGG pathways. It is noteworthy that the plasma components which are the main components during cell response to the intracellular or extracellular signals, such as the cell adhesion molecules are the most dysregulated molecules in $\mathrm{EC}$ as shown in the result of biological pathway analysis, the cellular component and KEGG pathway analyses (Fig. 3A, $\mathrm{C}$ and $\mathrm{D})$. Besides, the structure specific DNA binding components which may function during transcription and the oxidoreductase component which may be involved in cell metabolism and other processes are also dysregulated in EC (Fig. 3B).

Cell adhesion molecules are important for tissue integrity and also cell migration in tumor cells or other developmental cells $(16,21)$. E-cadherin, $\mathrm{N}$-cadherin and integrins which are the most famous adhesion molecules have been demonstrated to be associated with cell invasion of various cancer types (22-24), the transmembrane glycoprotein ALCAM (also known as CD166) which belongs to the immunoglobulin superfamily has been considered as the marker of breast cancer (25). In the 
A

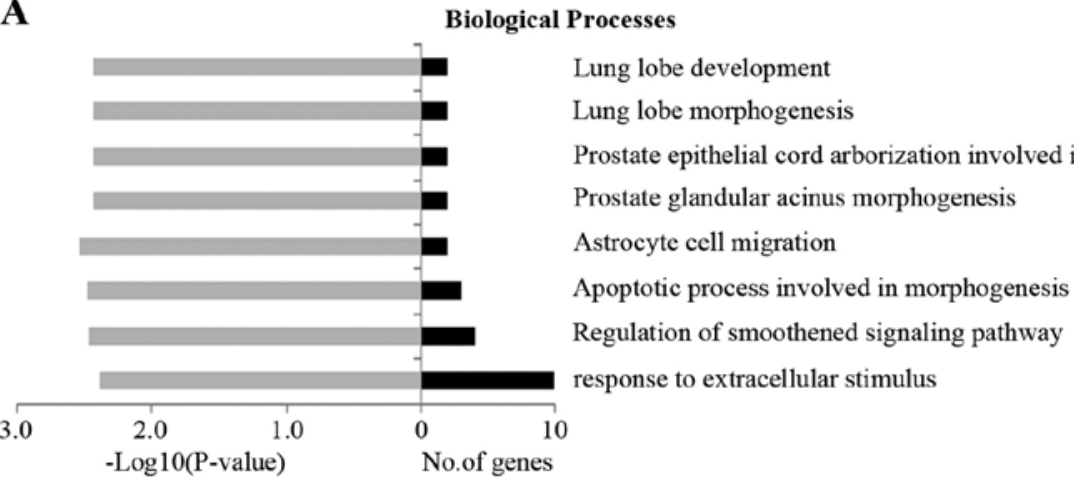

B

Molecular Functions

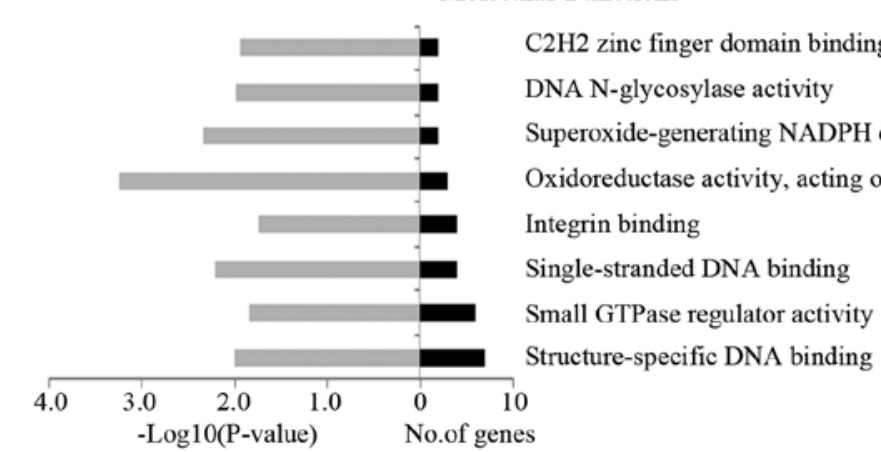

C

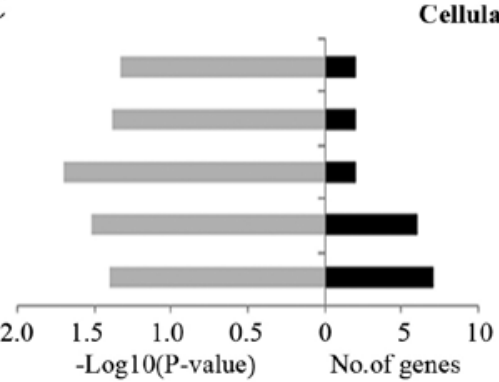

Synaptonemal complex

MHC protein complex

MHC class II protein complex

External side of plasma membrane

Side of membrane

D

KEGG

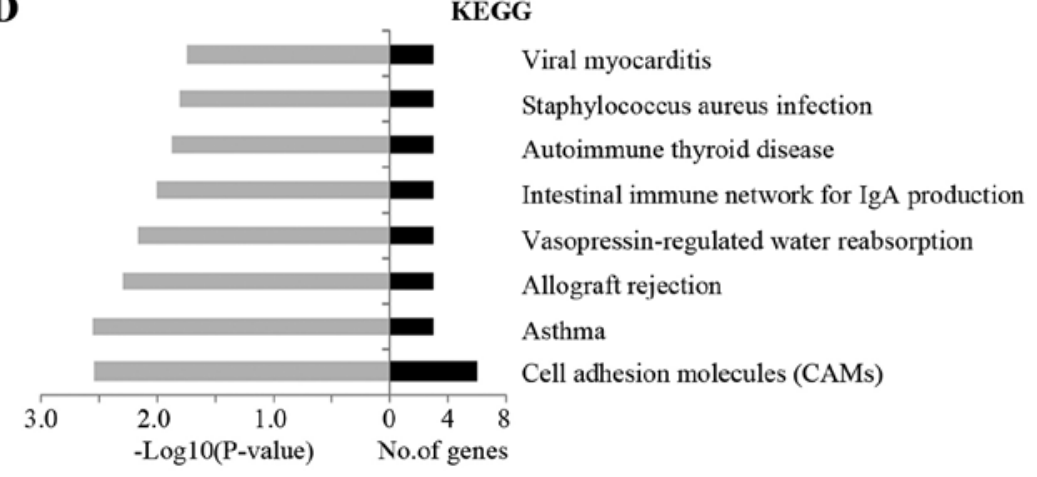

Figure 2. Distribution of differentially expressed lncRNAs. According to the NCBI database, the differentially expressed lncRNA (fold $>1.5$, P $<0.05$ ) include 62 intergenic (35 upregulated and 27 downregulated), 48 antisense (18 upregulated and 30 downregulated), 8 TEC (4 upregulated and 4 downregulated) and 54 other classes (such as processed transcript, sense overlapping or the lncRNAs whose classification are not very clear, 24 upregulated and 30 downregulated) (A). Most of the differentially expressed lncRNAs are 401-64000 bp in length (B). Chromosome distribution of the upregulated (C) and downregulated (D) IncRNAs show that the upregulated lncRNAs and the downregulated lncRNAs are distributed in different chromosomes.

present study, the cell adhesion molecules, such as ALCAM are also the most dysregulated mRNA.

mRNA-lncRNA co-expression network. IncRNA function has been implicated in several steps of carcinogenesis by acting as structural, catalytic or regulatory RNA or interacting with DNA, RNA and protein (16). However, only a few lncRNA functions have been elucidated. mRNA and IncRNA co-expression network, which can provide evidence for the involvement of new genes or lncRNAs in core biological functions, is very important for the prediction of the lncRNA function preliminarily $(19,26)$. Thus, we carried out the IncRNA and mRNA co-expression network in Guangzhou RiboBio Co. The co-expression network in Fig. 4A shows that ALCAM is co-expressed with 5 lncRNAs (ENST00000624279:RP11140I24, LOC102723552, KIAA0087, RP11-600K151 and 

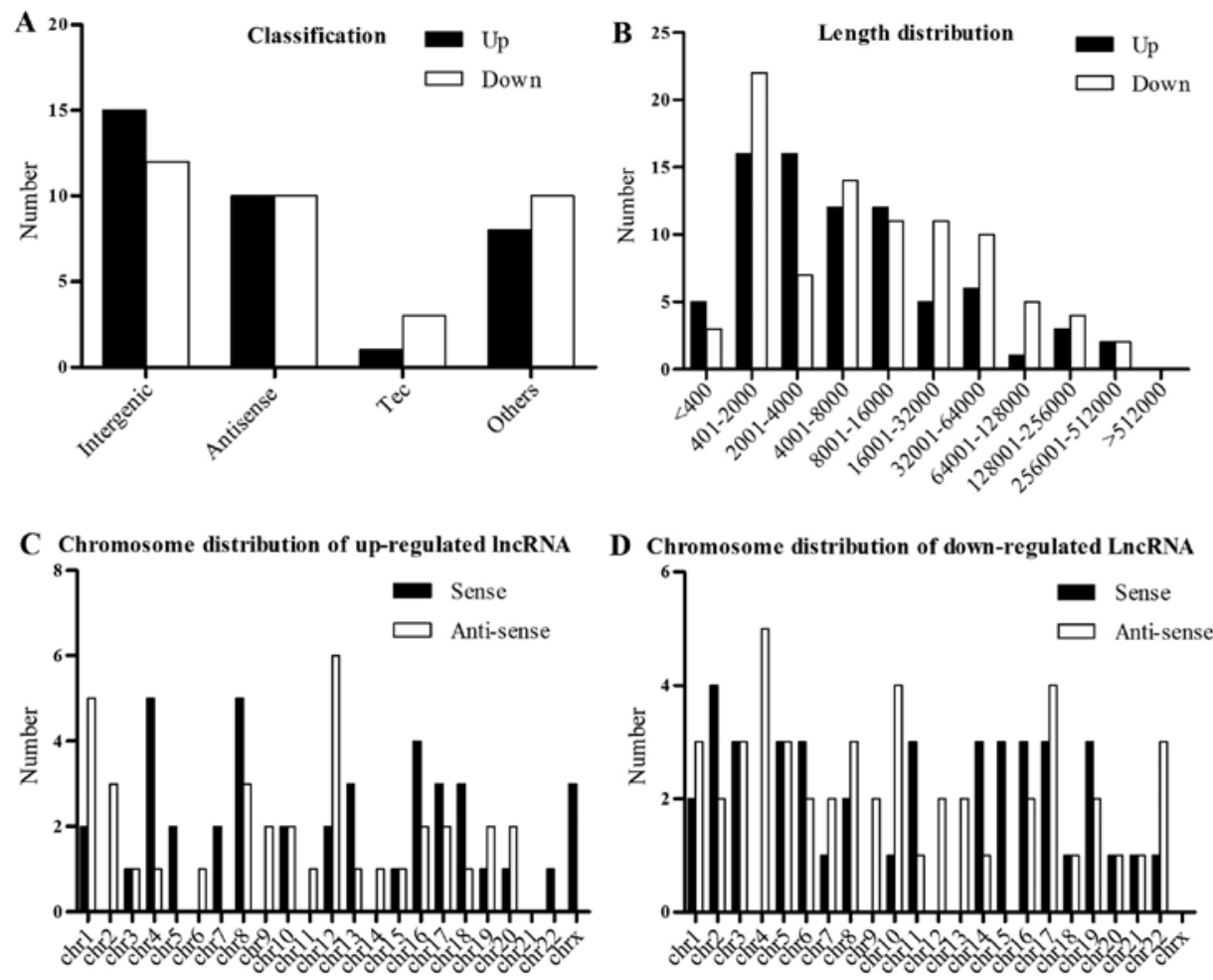

Figure 3. GO and KEGG analysis of differentially expressed lncRNAs. GO (A-C) and the KEGG pathway analysis (D) of the differentially expressed lncRNAs indicated that the plasma components such as the cell adhesion molecules which response to the intracellular or extracellular signals, the structure-specific DNA binding components as well as the oxidoreductases involved in metabolism are the most dysregulated molecules in EC compared to NE.
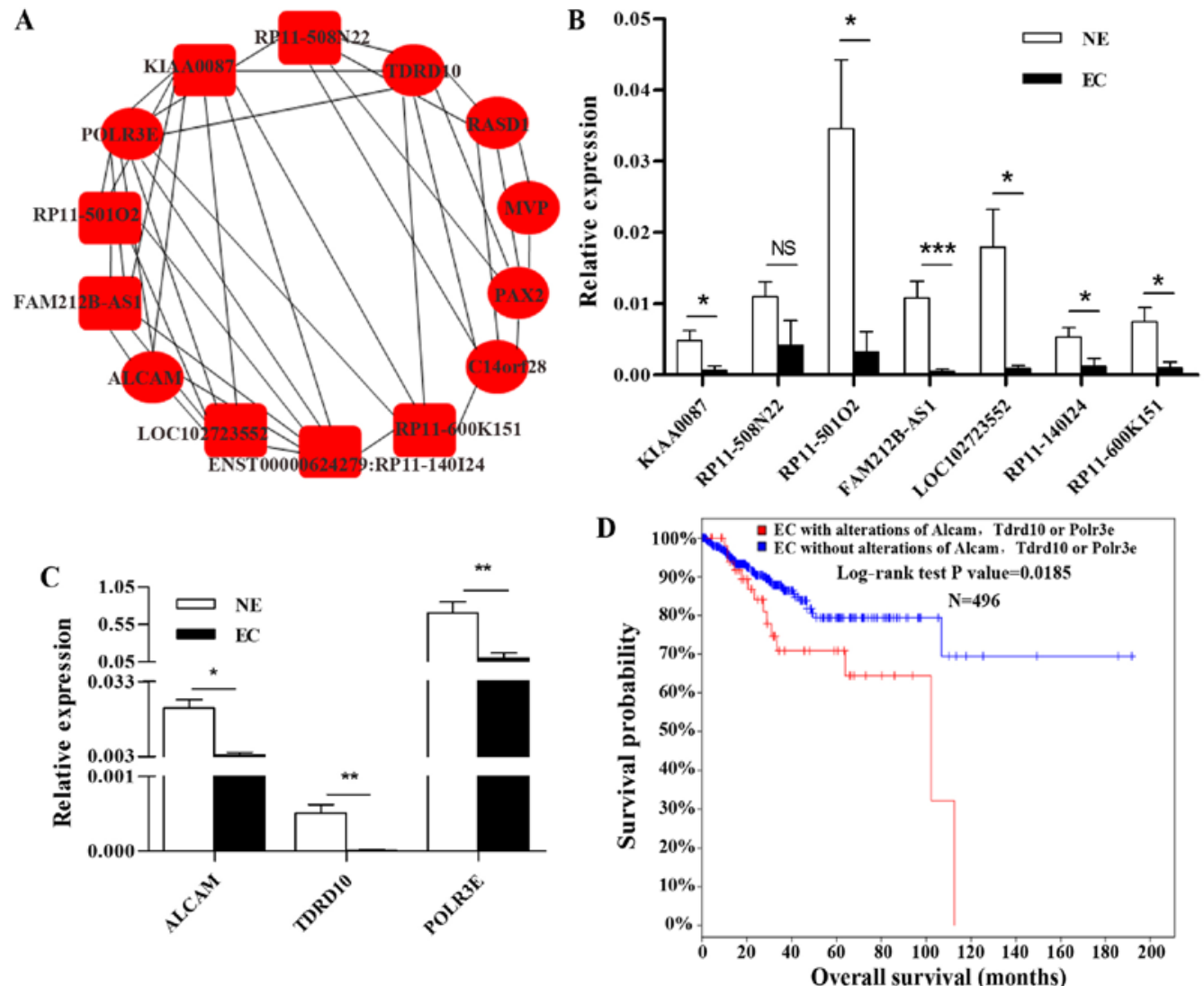

Figure 4. IncRNA and mRNA co-expression network showed a variety of co-expressed lncRNAs and mRNAs that are downregulated in EC. (A) A diagram of the differentially expressed lncRNA and mRNA co-expression network (red indicated the downregulated lncRNAs and mRNAs in EC). (B and C) Real-time PCR verification of those dysregulated lncRNAs (B) and mRNAs (C) that were co-expressed (fold $>5, P<0.05$ ). (D) Survival analysis of ALCAM, TDRD10 and POLR3E status shows divergent outcome of the gene altered cases (with lower overall survival rate) than the unaltered group according to the TCGA data presented in cBioPortal. 
FAM212B-AS1) and 1 mRNA (POLR3E), POLR3E is co-expressed with 6 lncRNAs (FAM212B-AS1, RP11-140I24, LOC102723552, RP11-508N22, RP11-501O2 and KIAA0087) and 2 mRNAs (ALCAM and TDRD10), TDRD10 is co-expressed with 3 lncRNAs (RP11-600K151, RP11-508N22 and KIAA0087) and 1 mRNA (POLR3E) which are differentially expressed in EC ( $>5$-fold, $\mathrm{P}<0.05)$. Real-time PCR verified that 6 of the 7 lncRNAs and all of the 3 mRNAs are dramatically downregulated in EC, which is consistent with the microarray data (Fig. 4B and C). Interestingly, most of these lncRNAs are relatively conserved between human, rhesus, rat and mouse as analyzed using UCSC website (http:// genome.ucsc.edu/) (data not shown).

The Cancer Genome Atlas (TCGA) database includes most of the tumor types and information on each case for community research use. cBioPortal for Cancer Genomics provides visualization and analysis of the TCGA datasets. Notably, the results presented in the cBioPortal of cancer genomics (http:// www.cbioportal.org/) showed that EC cases with ALCAM, TDRD10 and/or POLR3E alterations showed poorer overall survival rate as compared with cases without alterations of these genes (Fig. 4D).

\section{Discussion}

According to the ENCODE Project Consortium, the protein coding genes only account for $1 \%$ of the human genome (27). Most of the regions are not transcribed, such as a large class of the non-coding RNA. IncRNA functions as structural, catalytic, regulatory RNAs have been found to be involved in a variety of processes, such as development (18), tumorigenesis (16), cardiovascular disease (28) and neurodegenerative diseases (29). Similarly to the protein coding genes, lncRNAs which are distributed in the cytoplasm, nucleus and also transported to the extracellular matrix can be identified as the biomarkers of various diseases. For example, studies found that lncRNADANCR can be used as a prognostic biomarker of hepatocellular carcinoma (30). The circulating lncRNA-LIPCAR can be used to predict survival of patients with heart failure (31). IncRNAs are also known as biomarkers or therapeutic target of cancers, such as breast (32), lung (33) and ovarian cancer (19). Since most of the lncRNAs function through the regulation of the mRNAs the IncRNA-mRNA co-expression network provides us with a powerful platform for the prediction of the lncRNA function (19). For example, RP11-284N8.3.1 and AC104699.1.1 which were identified using the lncRNA-mRNA co-expression network are found to be the independent predictive biomarkers of ovarian cancer (19). In this study, we first used microarray analysis to find the differentially expressed lncRNAs in EC, and then by using GO and KEGG as well as the IncRNA-mRNA co-expression network, we predicted that the 6 lncRNAs and 3 mRNAs are probably the early biomarkers of EC.

The glycolysis which produces ATP more quickly is strongly enhanced in tumor cells $(34,35)$. Here, we used the GO analysis to prove that the main molecular function involved is the oxidoreductase activity, emphasizing the difference between the metabolism between the tumor and normal tissue (Fig. 3B).

The adhesion molecules have been demonstrated to be the important regulators of endometrial epithelium integrity, its decreased expression will disrupt the integrity of the NE and may be involved in the pathology of the endometrium (22). Asthma which affects $5-10 \%$ of the population may increase the patients' risk of cancer is also altered in EC as compared with control (36). The chronic inflammatory disease associated gene has been found to help cancers metastasize and may be the predictor of brain cancer in children $(37,38)$. Notably, the KEGG analysis showed that the adhesion molecules and asthma associated genes are the top two differentially altered pathways in EC.

Endometrial carcinoma which is a common malignancy of female reproductive tract accounts for $\sim 1-2 \%$ of the tumor deaths in the western world (1). It is also becoming more prevalent in China each year. Thus, it is very urgent for us to find the predictive biomarkers of EC. Here, in order to find whether lncRNAs can be used as EC biomarkers, we performed the lncRNA microarray. By using the microarray data and the lncRNA-mRNA co-expression network, we found 6 lncRNAs (KIAA0087, RP11-501O2, FAM212B-AS1, LOC102723552, RP11-140I24 and RP11-600K151) and 3 mRNAs (ALCAM, TDRD10 and POLR3E) that are functionally related in EC. Interestingly, ALCAM is highly expressed in the endometrium glandular cells and its expression was decreased in $\sim 50 \%$ of the endometrial cancer tissues while POLR3E is moderately expressed in the endometrium glandular cells and its expression was decreased in $\sim 50 \%$ of EC according to the human protein atlas website which is consistent with our data (http:// www.proteinatlas.org/). The TCGA data also confirmed that these three gene alterations indicated poorer overall survival as compared with the cases without these gene alterations. We also validated that the IncRNAs which are co-expressed with these three mRNAs are downregulated in EC.

Since the mechanism of the type I EC and type II EC is quite different, distinguish the different type of $\mathrm{EC}$ is quite important. Different from the differentially expressed lncRNAs between the randomly selected EC samples and the adjacent non-tumor tissue reported before (20), we reported the differentially expressed lncRNAs in type I EC as compared with NE using the microarray and the lncRNAs and mRNA co-expression network for the first time. The differentially expressed lncRNAs may be indicators of the type I EC and may provide the potential information for the evaluation of EC.

\section{Acknowledgements}

The present study was financially supported by the National Natural Science Foundation of China (81302304 and 81572556) and the Key Program of Science and Technology Development Fund of Nanjing Medical University (2013NJMU145, 2014NJMU103, 2014NJMU098 and 2015NJMUZD063).

\section{References}

1. Plataniotis G and Castiglione M; ESMO Guidelines Working Group: Endometrial cancer: ESMO Clinical Practice Guidelines for diagnosis, treatment and follow-up. Ann Oncol 21 (Suppl 5): $\mathrm{v} 41-\mathrm{v} 45,2010$.

2. Chen W, Zheng R, Baade PD, Zhang S, Zeng H, Bray F, Jemal A, Yu XQ and He J: Cancer statistics in China, 2015. CA Cancer J Clin 66: 115-132, 2016.

3. Bokhman JV: Two pathogenetic types of endometrial carcinoma. Gynecol Oncol 15: 10-17, 1983 
4. Weiderpass E, Persson I, Adami HO, Magnusson C, Lindgren A and Baron JA: Body size in different periods of life, diabetes mellitus, hypertension, and risk of postmenopausal endometrial cancer (Sweden). Cancer Causes Control 11: 185-192, 2000.

5. Yasa C, Takmaz O, Dural O and Akhan SE: The value of tumor markers in endometrial carcinoma: Review of literature. J Cancer Ther 4: 966-970, 2013.

6. Dudley B, Brand RE, Thull D, Bahary N, Nikiforova MN and Pai RK: Germline MLH1 mutations are frequently Identified in Lynch Syndrome patients withcolorectal and endometrial carcinoma demonstrating isolated loss of PMS2 immunohistochemical expression. Am J Surg Pathol 39: 1114-1120, 2015.

7. Vasen HF, Stormorken A, Menko FH, Nagengast FM, Kleibeuker JH, Griffioen G, Taal BG, Moller P and Wijnen JT: MSH2 mutation carriers are at higher risk of cancer than MLH1 mutation carriers: A study of hereditary nonpolyposis colorectal cancer families. J Clin Oncol 19: 4074-4080, 2001.

8. Basil JB, Swisher EM, Herzog TJ, Rader JS, Elbendary A, Mutch DG and Goodfellow PJ: Mutational analysis of the PMS2 gene in sporadic endometrial cancers with microsatellite instability. Gynecol Oncol 74: 395-399, 1999.

9. Devlin LA, Graham CA, Price JH and Morrison PJ: Germline MSH6 mutations are more prevalent in endometrial cancer patient cohorts than hereditary non polyposis colorectal cancer cohorts. Ulster Med J 77: 25-30, 2008.

10. Wiegand KC, Shah SP, Al-Agha OM, Zhao Y, Tse K, Zeng T, Senz J, McConechy MK, Anglesio MS, Kalloger SE, et al: ARID1A mutations in endometriosis-associated ovarian carcinomas. N Engl J Med 363: 1532-1543, 2010.

11. Albitar L, Carter MB, Davies S and Leslie KK: Consequences of the loss of p53, RB1, and PTEN: Relationship to gefitinib resistance in endometrial cancer. Gynecol Oncol 106: 94-104, 2007.

12. Swisher EM, Peiffer-Schneider S, Mutch DG, Herzog TJ, Rader JS, Elbendary A and Goodfellow PJ: Differences in patterns of TP53 and KRAS2 mutations in a large series of endometrial carcinomas with or without microsatellite instability. Cancer 85: 119-126, 1999.

13. Senol S, Sayar I, Ceyran AB, Ibiloglu I, Akalin I, Firat U, Kosemetin D, Engin ZP and Aydin A: Stromal clues in endometrial carcinoma: Loss of expression of beta-catenin, epithelial-mesenchymal transition regulators, and estrogenprogesterone receptor. Int J Gynecol Pathol 35: 238-248, 2015.

14. Kafshdooz T, Mohaddes Ardabili SM, Kafshdooz L, Tabrizi AD, Ghojazadeh M, Gharesouran J and Akbarzadeh A: C-kit mutations in endometrial cancer: Correlation with tumor histologic type. Asian Pac J Cancer Prev 16: 7449-7452, 2015.

15. Zighelboim I, Schmidt AP, Gao F, Thaker PH, Powell MA, Rader JS, Gibb RK, Mutch DG and Goodfellow PJ: ATR mutation in endometrioid endometrial cancer is associated with poor clinical outcomes. J Clin Oncol 27: 3091-3096, 2009.

16. Yang G, Lu X and Yuan L: LncRNA: A link between RNA and cancer. Biochim Biophys Acta 1839: 1097-1109, 2014.

17. Lv M, Xu P, Wu Y, Huang L, Li W, Lv S, Wu X, Zeng X, Shen R, Jia X, et al: LncRNAs as new biomarkers to differentiate triple negative breast cancer from non-triple negative breast cancer. Oncotarget 7: 13047-13059, 2016.

18. Herriges MJ, Swarr DT, Morley MP, Rathi KS, Peng T, Stewart KM and Morrisey EE: Long noncoding RNAs are spatially correlated with transcription factors and regulate lung development. Genes Dev 28: 1363-1379, 2014.

19. Guo Q, Cheng Y, Liang T, He Y, Ren C, Sun L and Zhang G: Comprehensive analysis of IncRNA-mRNA co-expression patterns identifies immune-associated lncRNA biomarkers in ovarian cancer malignant progression. Sci Rep 5: 17683, 2015.

20. Zhai W, Li X, Wu S, Zhang Y, Pang H and Chen W: Microarray expression profile of IncRNAs and the upregulated ASLNC04080 lncRNA in human endometrial carcinoma. Int J Oncol 46: $2125-2137,2015$
21. Singh $\mathrm{H}$ and Aplin JD: Adhesion molecules in endometrial epithelium: Tissue integrity and embryo implantation. J Anat 215: 3-13, 2009.

22. Avizienyte E, Wyke AW, Jones RJ, McLean GW, Westhoff MA, Brunton VG and Frame MC: Src-induced de-regulation of E-cadherin in colon cancer cells requires integrin signalling. Nat Cell Biol 4: 632-638, 2002.

23. Park JH, Lee BI, Song ES, Whang SO, Lee WY and Cho SJ: Hypermethylation of $\mathrm{E}$-cadherin in endometrial carcinoma. J Gynecol Oncol 19: 241-245, 2008.

24. Hecht JL, Dolinski BM, Gardner HA, Violette SM and Weinreb PH: Overexpression of the alphavbeta6 integrin in endometrial cancer. Appl Immunohistochem Mol Morphol 16: 543-547, 2008

25. Kulasingam V, Zheng Y, Soosaipillai A, Leon AE, Gion M and Diamandis EP: Activated leukocyte cell adhesion molecule: A novel biomarker for breast cancer. Int J Cancer 125: 9-14, 2009.

26. Stuart JM, Segal E, Koller D and Kim SK: A gene-coexpression network for global discovery of conserved genetic modules. Science 302: 249-255, 2003.

27. Birney E, Stamatoyannopoulos JA, Dutta A, Guigó R, Gingeras TR, Margulies EH, Weng Z, Snyder M, Dermitzakis ET, Thurman RE, et al; Children's Hospital Oakland Research Institute: Identification and analysis of functional elements in $1 \%$ of the human genome by the ENCODE pilot project. Nature 447: 799-816, 2007.

28. Congrains A, Kamide K, Oguro R, Yasuda O, Miyata K, Yamamoto E, Kawai T, Kusunoki H, Yamamoto H, Takeya Y, et al: Genetic variants at the 9p21 locus contribute to atherosclerosis through modulation of ANRIL and CDKN2A/B. Atherosclerosis 220: 449-455, 2012.

29. Johnson R: Long non-coding RNAs in Huntington's disease neurodegeneration. Neurobiol Dis 46: 245-254, 2012.

30. Yuan SX, Wang J, Yang F, Tao QF, Zhang J, Wang LL, Yang Y, Liu H, Wang ZG, et al: Long noncoding RNA DANCR increases stemness features of hepatocellular carcinoma by derepression of CTNNB1. Hepatology 63: 499-511, 2015.

31. Kumarswamy R, Bauters C, Volkmann I, Maury F, Fetisch J, Holzmann A, Lemesle G, de Groote P, Pinet F and Thum T: Circulating long noncoding RNA, LIPCAR, predicts survival in patients with heart failure. Circ Res 114: 1569-1575, 2014

32. Lei B, Xu SP, Liang XS, Li YW, Zhang JF, Zhang GQ and Pang D: Long non-coding RNA MVIH is associated with poor prognosis and malignant biological behavior in breast cancer. Tumour Biol 37: 5257-5264, 2015.

33. Gutschner T, Hämmerle M, Eissmann M, Hsu J, Kim Y, Hung G, Revenko A, Arun G, Stentrup M, Gross M, et al: The noncoding RNA MALAT1 is a critical regulator of the metastasis phenotype of lung cancer cells. Cancer Res 73: 1180-1189, 2013.

34. Chang CH, Qiu J, O'Sullivan D, Buck MD, Noguchi T, Curtis JD, Chen Q, Gindin M, Gubin MM, van der Windt GJ, et al: Metabolic competition in the tumor microenvironment is a driver of cancer progression. Cell 162: 1229-1241, 2015.

35. Raccosta L, Fontana R, Corna G, Maggioni D, Moresco M and Russo V: Cholesterol metabolites and tumor microenvironment: The road towards clinical translation. Cancer Immunol Immunother 65: 111-117, 2015.

36. Ji J, Shu X, Li X, Sundquist K, Sundquist J and Hemminki K: Cancer risk in hospitalised asthma patients. Br J Cancer 100: 829-833, 2009

37. Roncarolo F and Infante-Rivard C: Asthma and risk of brain cancer in children. Cancer Causes Control 23: 617-623, 2012.

38. Elinav E, Nowarski R, Thaiss CA, Hu B, Jin C and Flavell RA: Inflammation-induced cancer: Crosstalk between tumours, immune cells and microorganisms. Nat Rev Cancer 13: 759-771, 2013. 